\title{
Research on Identification Methods of Civil Engineering Structures
}

\author{
Ping Gan ${ }^{1, ~ a ~}$, Zhizhang Wan ${ }^{1, b}$ \\ ${ }^{1}$ Nanchang Institute of Science \& Technology, Nanchang, 330108, China

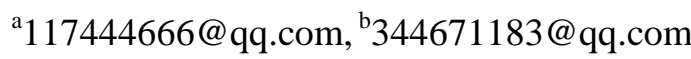

Keywords: civil engineering structures; identification methods; damage diagnosis

\begin{abstract}
At present, our country's economic construction is developing rapidly, and the country's investment in urban infrastructure construction is increasing. The comfort and speed of the new type of architecture, and the structural loss of the building have entered our sight. This paper analyzes the common identification methods of civil engineering structures and looks forward to the future trend of structural identification methods for civil engineering to provide some references for the relevant researchers.
\end{abstract}

\section{Introduction}

Structural health monitoring is based on on-site nondestructive sensing technology and structural characteristics analysis methods to detect and reveal the process of structural degradation or damage [1]. Structural health monitoring is an online technology. It can make real-time assessment of structural status by monitoring data and can also make real-time diagnosis of structural integrity rapidly after earthquake and hurricane. Therefore, to obtain the relevant state information through the structural health monitoring technology for design validation and engineering construction to provide strong technical support and control, safe operation and maintenance decision, it is very important to the evaluation of the structure process of life safety, integrity, durability and applicability. A complete structural health monitoring system mainly includes: sensor and signal acquisition system, data transmission and signal processing system, structural damage identification and model correction system, structural health diagnosis and safety evaluation system. Among them, structural damage identification and model correction system are of great significance to structural health diagnosis and safety evaluation. Therefore, it can be said that the theory and method of structural damage identification and model correction is one of the core contents of the research of structural health monitoring. In recent years, the dynamic characteristics of the structure observation of the damage identification because it can evaluate the overall structure, cost-effectiveness, easy online implementation based on advantages, and become one of the most important methods for the overall evaluation of structure technology is a hot and difficult problem in structural damage identification and health diagnosis research. These methods of diagnosis and identification will become more and more complex with the structure of civil engineering and lose the efficiency and accuracy of detection. Some detection methods belong to the local damage detection technology of civil engineering structure, which is not suitable for the detection of the damage of the whole engineering structure, which leads to the limitation of the application [2].

\section{Common Identification Methods of Civil Engineering Structures}

\subsection{Index Identification Method}

The method is mainly based on establishing a dynamic data model to transform the data obtained from the original static data model, to get the vibration response data. Then, the data is compared with the original theoretical data to evaluate whether all the mechanical parameters of the civil engineering structure meet the standard or meet the safety requirements of the structure. This model updating and 
index recognition diagnosis method is more accurate and fast than the traditional static parameter diagnosis method, and it can judge the damage degree and safety performance parameters of civil engineering structure. The key of this model is the establishment of models and the comparison of data, so it will apply to many mathematical models, which requires our detection and diagnosis personnel to have higher professional knowledge and skills. Of course, for a large civil engineering project, its safety diagnosis and evaluation are a very important matter, so the work of diagnosing and inspecting personnel is particularly important. We must carry on the effective recognition of civil engineering structure vibration damage, must be measured in civil engineering, master the test data in detail based on data analysis, the mechanism of vibration engineering damage identification in civil engineering, but the vibration of structural damage during the test, the test data on the impact of the incomplete to effectively identify injuries. The test mode is incomplete and the degree of freedom of the test is incomplete. These two situations cause the modal mismatch between the modal and the analysis model of the test to affect the effect of the analysis, which results in the mismatch between the degree of freedom of the test and the freedom of the analysis model, and the effect of the analysis. Test in some degrees of freedom, very hard to turn the relevant data, so the degree of freedom, freedom is not a complete test of objective problems, it is very difficult to avoid, these problems lead to incomplete test data [3].

\subsection{Genetic Computing Method}

Genetic computing is an interdisciplinary engineering structure damage detection method developed in recent years. The genetic algorithm can analyze the safety parameters of the engineering structure as much as possible when the dynamic parameters are less than the model. Genetic algorithm only needs to calculate the feasible solution of the target, does not need continuity of objective function, optimizes parallel search and multiple cue, and obtains the most effective result using less data analysis. Because of the small number of data needed, the application of genetic computing method is wide and the anti-interference ability is strong, it is very popular with the technical personnel of the civil engineering structure damage diagnosis. According to the modal information, these methods can be further divided into natural frequency, mode of vibration, strain mode, modal strain energy, flexibility, frequency response function and dynamic residual vector. In the method based on modal domain data, using sensitivity analysis or model updating methods can damage localization and quantification, but requires a baseline finite element model of intact structure, finite element model of the damage identification accuracy depends on the accuracy of the method established by the modal parameters; direct or indirect comparison before and after damage usually only damage detection and localization, does not need the finite element model of the structure is obtained; and the damage identification method of pattern recognition and neural network based on the type and number of relevant training samples. Genetic algorithm is a method based on natural selection and natural genetic mechanism optimization, the optimization method of damage assessment, access to information in the test are few, can quickly determine the location and extent of damage, even if the modal information lost, genetic algorithm optimization ability will not be affected. Genetic algorithm only needs to calculate the target value of each feasible solution instead of the continuity of objective function. It does not need gradient information and adopts multi search parallel search method to optimize it, so it will not fall into local minimum, and is easy to use and robust [4].

\subsection{Wavelet Analysis Method}

Wavelet analysis method is the latest achievements of analysis technology development research on harmonic mathematical theory in recent years, the new signal processing method can well deal with civil engineering structure in the anti-vibration problem, making the original those in analysis of actual engineering using limited dynamic parameter analysis method has been expanded. Using the characteristics of strong anti-interference ability of wavelet analysis, the defects of missing and attenuated signal in the process of model data transfer can be eliminated well. The method of wavelet signal processing is very useful for the recognition and analysis of positive and negative signals. On 
the one hand, the interference of the reverse signal can be eliminated by wavelet analysis. On the other hand, wavelet analysis can discriminate the small difference between the positive and negative signals, to distinguish them well. The detection method of wavelet analysis is a good method to detect the damage of vibration in civil engineering structure. Based on the different functions of wavelet transform, wavelet transform can be further divided into wavelet singularity detection method, wavelet transform coefficient transformation method based on damage and wavelet transform and elastic wave propagation theory. If the acquiring dynamic structure point measured in the response information is divided into three categories: time domain information in frequency domain information and spatial information, then, using the method of time domain data based only on the time axis information; using the method of modal domain data based only on the frequency axis information. The method based on wavelet analysis can make full use of the information on the time frequency plane of the response. Therefore, such methods can not only locate the damage, but also provide the time information of the damage.

\subsection{Dynamic Parameter Method}

The so-called dynamic parameters are compared with static parameters. Static parameters are only suitable for judging the local damage degree of civil engineering. At present, the dynamic parameters include frequency, mode of vibration, modal curvature, frequency response function, strain mode, modal flexibility matrix, transfer function, power spectrum, energy transfer ratio and modal assurance criterion. These dynamic parameters will change with the change of the position of the processing object, so after the local damage detection of engineering structures, change the position, these dynamic parameters will also change, so the dynamic parameters of different local will have a certain relationship, finally through the analysis of model parameters can be obtained damage diagnosis the results of the civil engineering structure. The dynamic response and the amount of its derivatives, which are obtained from the dynamic test, are called power fingerprints. The dynamic fingerprint method is to determine the real state of the structure by analyzing the dynamic fingerprint changes associated with the dynamic characteristics of the structure. Once the structure is damaged, its structural parameters, such as stiffness, mass and damping, will change, resulting in the change of the corresponding dynamic fingerprints. The changes in these dynamic fingerprints be a sign of structural damage to diagnose structural damage. These calculations do not constitute a serious obstacle, however, for large-scale structural optimization problems, many finite element analysis and calculation will be a heavy burden. Therefore, how to effectively reduce the amount of finite element analysis and calculation is the key to the successful application of the algorithm to the optimization design of large structures. In the same way, for structural damage identification, reducing the amount of calculation is an important problem that must be considered when using the algorithm to solve the problem.

\subsection{Neural Network Method}

As a new model of neural network diagnosis method and applied to the diagnosis of damage detection of civil engineering structures, in the data analysis and transmission process, is the neural basis of unit, so analyze and store the data in this model are very fast and efficient. In addition, by dealing with the data model of neural network, the fault tolerance of every index data of engineering structure damage will increase greatly, which is very helpful for model updating and index recognition. This method usually uses the characteristics of the structural vibration response in the local time domain or the statistical characteristics in a time domain to identify the damage of the structure. Most of these methods are based on the time series analysis model. Besides, there are also some ways to identify the physical parameters of the structure by using the extended filtering method. By comparing the physical parameters and the intact state of the structure at present, we can identify the damage. These methods directly use the time domain data of test, do not need to make a variety of transformations, and the damage related features in the response signal will not be distorted or filtered due to data transformation. The inversion method based on the frequency observation mainly uses the analytic method and the numerical method of sensitivity analysis. The study shows that it is difficult 
to use sensitivity method to identify structural damage when the number of observed modes is less than the damage parameters. The reason for this difficulty is that the system is obviously underdetermined, and there is not enough independent information to determine all stiffness reduction coefficients. In this case, the solution obtained by using generalized inverse will become ill. The drawback is that some signal characteristics related to injury may be concealed or overwhelmed by the characteristics which are not related to the damage. If the excitation source changes or the environmental state changes, it will be difficult to identify the damage with this method.

\section{Outlook of Identification Method of Civil Engineering Structures}

The application of new self-sensing intelligent materials may also greatly promote the development of structural damage identification technology. At present, many advanced into service and the new major structures already installed real-time health monitoring system, according to the characteristics of detection structure and detect target, choose several effective methods for damage identification can be implemented, the establishment of health monitoring system can be integrated into the damage identification results of various methods combined damage identification module and in the structural damage identification methods play as an important way in practical engineering. In this way, the advantages of various methods can be set up to get more reliable results of damage identification. For example, the development of fusion algorithm structure damage identification of partial response and overall response; method of modal domain data and method based on wavelet analysis based on combining the method of modal or domain data based on the location of damage to a range, again by the wavelet space domain singularity detection method based on the damage location. For the damage identification methods that need finite element models with good structural integrity, for example, based on dynamic residual vectors, model updating, sensitivity analysis and pattern matching, establishing finite element models with certain accuracy is the key to make such methods effective. Therefore, it is very important to carry out dynamic test for the newly built structure and to measure the measured response data well, and it is also necessary to develop more effective and practical model updating techniques to get the finite element models that can reflect the dynamic characteristics of the real structure better. Most of the current researches and applications are for simple components, such as beam or panel type. For complex structures, the application of substructure vibration analysis method is of great significance for promoting the practical application of structural damage identification. For example, for extremely complex structures, damage identification can be divided into several sub structures, identifying damage on each sub structure respectively, which not only improves the recognition efficiency, but also reduces the number of sensors.

\section{Conclusion}

The damage identification and diagnosis of various complex engineering structures in the process of use should be further studied for many problems. Due to the less application of damage identification and diagnosis in engineering structures, various damage tests for different engineering structures must be carried out, and methods of identification and detection should be verified, so that these methods can be widely applied.

\section{References}

[1] Zhang Wei. Graphics Digitizer Technology in Civil Engineering Structures Test of Inquiry [J]. 2016, 43(11): 112-113.

[2] Zhang Yongkang. The construction technology of concrete structure in civil engineering [J]. Shanxi Architecture, 2017, 43(24): 95-96.

[3] Jia Lixin. Construction technology anaylisis of concrete structure civil engineering building [J]. China Standardization, 2016(17): 185. 
[4] Ren Haiying. Discussion on Steel Structure Technology in Civil Engineering Construction [J]. Architectural Knowledge, 2017(7): 67. 